\title{
RECURSOS NATURALES Y LA CONTABILIDAD AMBIENTAL
}

\author{
NATURAL RESOURCES AND ENVIRONMENTAL ACCOUNTING \\ Elsa Esther Choy Zevallos* \\ Docente Asociada de la Facultad de Ciencias Contables \\ Universidad Nacional Mayor de San Marcos-UNMSM / Lima-Perú \\ [Recepción: Julio de 2013/ Conformidad: Agosto 2013]
}

\section{RESUMEN}

El trabajo tiene como propósito describir aquellos recursos naturales que se identifican como activos ambientales y no se reconocen como tal, en la contabilidad financiera tradicional. Algunas de las razones indican que éstos son consideradas de libre disponibilidad y carecen de precio, al no existir un mercado definido para sus transacciones.

La necesidad de valorar los recursos naturales y la normativa contable ambiental, son algunos temas importantes que se desarrollan en este trabajo, como un avance parcial, debido a su complejidad y limitación de información, siendo conscientes que se requiere aún mayor investigación empírica.

\section{Palabras Clave:}

Contabilidad ambiental, recursos naturales, valor económico

\section{ABSTRACT}

These work aims to describe those who identify natural resources and environmental assets that are not recognized as such in the traditional financial accounting. Some reasons indicate that they are considered unrestricted and lack of value in the absence of a defined market for their transactions.

The need to value natural resources and environmental accounting regulations are some important topics developed in this work, as a partial breakthrough, due to its complexity and limited information, being aware that more empirical research is required.

\section{Keywords:}

Environmental, accounting, natural resources, economic value.

* Doctora en Ciencias Contables y Empresariales. Magister en Dirección Financiera. Contadora Pública Colegiada Certificada. Docente Investigadora-UNMSM. E mail: eschoyz@outlook.com 


\section{INTRODUCCIÓN}

La abundancia de nuestros recursos naturales y el poco entendimiento de las propiedades y funciones de los ecosistemas, no han permitido desarrollar un sistema de precios de mercado. Sin embargo, bajo el concepto de desarrollo sostenible emerge la necesidad de valorar estos recursos naturales que han sido excluidos en la toma de decisiones políticas.

Si bien, se cuenta con avances metodológicos en la valorización económica de los recursos naturales; son pocos los estudios que profundizan a este nivel de investigación, lo cual podría atribuirse a la complejidad en las metodologías sobre valoración económica ambiental o la controversia que todavía éstas suscitan.

Por otro lado, la contabilidad que se encarga de registrar el patrimonio de una entidad y su valor agregado, debe ocuparse de registrar los recursos naturales a fin de reflejarlos en las cuentas nacionales.

El sector académico juega un papel prioritario desde los centros de educación superior como la Facultad de Ciencias Contables de la Universidad Nacional Mayor de San Marcos, donde de manera directa $o$ indirectamente y gracias a las políticas y estrategias planteadas por nuestra universidad, la investigación e innovación, así como, los procesos de capacitación que se desarrollan de forma integrada y multidisciplinaria, generan mayor conocimiento que potencia el desempeño ambiental.

\section{RECURSOS NATURALES Y CONTABILIDAD AMBIENTAL}

Reconocer que los recursos naturales del planeta son finitos y que están sujetos al agotamiento, se les ubica en la categoría de "recurso escaso".

Si se pretende establecer su valor económico, es necesario analizar el concepto de riqueza; Fisher lo separa en bienes de capital y en valor de capital; el primero los concibe como la riqueza contenida en el stock de capital producido y en la tierra; mientras el valor de capital es la valuación de los flujos que genera esa riqueza a lo largo del tiempo.

El stock de capital producido, conocido también como activos fijos, tales como: maquinaria, edificios y otros; son aquellos que contribuyen a la producción de bienes y servicios con la transformación de materias primas, provienen de otros procesos productivos y a través del tiempo, pierden funcionalidad ya sea por el desgaste u obsolescencia.

La tierra, es un elemento cuya definición debe adecuarse a nuestros tiempos. Fisher, la entendía como la generadora de productos agrícolas en las plantaciones de madera en los bosques y de minerales en las minas. Pero, al reconocerse otros recursos como el aire; agua dulce para el consumo; el término tierra se complementaría como recursos del medio ambiente.

Con el reconocimiento de estos dos elementos aparece la necesidad de medirlos a partir de la creación de unidades similares que permitan su comparación y que ayuden a su intercambio en el giro normal del mercado. Surge la necesidad de establecer una valuación para representar el valor económico de cada uno de ellos, imperando la unidad monetaria para estandarizar un lenguaje que permita calcular una cifra representativa del capital.

Asimismo, el concepto de contabilidad recoge la necesidad de describir cuantitativamente la riqueza con sus agregados y la circulación del ingreso preveniente de lo producido por los stocks de capital y la tierra. De esta forma, se puede justificar que la contabilidad tiene que ocuparse de los aspectos ambientales para reflejarlos en los informes de los entes económicos.

Con esta explicación es posible comprender muchos aspectos de la economía a nivel macroeconómico o microeconómico, aún cuando existan muchas variables que no pueden ser medidas de esta forma.

\section{CONTABILIDAD MACROECONÓMICA}

Como se explicó, la unidad monetaria no refleja realmente la situación del medio ambiente, pero si refleja la situación financiera de la economía. Por tanto, es necesario buscar un mecanismo que permita hacer un acercamiento en términos de unidades de medida diferentes para reconocer esta situación. 
La Organización de las Naciones Unidas (ONU) plantea a este problema, una solución a nivel de la contabilidad nacional (Cuentas Nacionales) y lo plasma en el Manual llamado Sistema de Contabilidad Ambiental y Económica Integrada. Este manual plantea una estructura de cuentas satélites compuestas por matrices macroeconómicas donde se refleja la información de la relación entre la economía y el medio ambiente en unidades como volumen, energía, etc. y luego, la compara con la información macroeconómica recogida en unidades monetarias.

La solución a los problemas del reconocimiento de materiales y de la valuación son las dos preocupaciones que se plantea a partir de la metodología de cuentas satélites utilizadas en la contabilidad macroeconómica para los sistemas de cuentas nacionales. Estas se proponen como solución a la incapacidad del marco central de las cuentas nacionales para tratar temas específicos como la educación, el transporte o el medio ambiente y consiste en un sistema de cuentas anexo a la Contabilidad Nacional, su elaboración no afecta de ninguna manera el marco central, al contrario, deben responder a los datos mostrados en el mismo respetando su contenido. Así este tipo de cuentas toma la información del marco central, la clasifica y ordena de manera particular para revelar datos de un tema específico.

\section{CONTABILIDAD MICROECONÓMICA}

La contabilidad ambiental necesita de un soporte contable que sirva como modelo a cualquier empresa. Los principios contables:

Moneda o común denominador, es un principio básico que busca que las cifras contenidas en los estados contables muestren de manera confiable y uniforme, la realidad económica y financiera de los entes. Significa que la contabilidad financiera tiene sólo la capacidad de reflejar las transacciones o realidades que sean susceptibles de medición monetaria. Sin embargo, algunas situaciones ambientales del accionar organizacional son de difícil cuantificación y valuación monetaria, es por eso que se hace necesario abordar los aspectos de medición y valuación desde una perspectiva amplia que incluya, cuantificaciones en unidades físicas.

Según este supuesto, debe existir un conjunto de valores activos expresados en una unidad monetaria. Pero, como los flujos físicos se cuantificaron en unidades de cantidad, estas unidades reemplazaron los nombrados valores.

Ciertamente el modelo contable ambiental de flujos físicos debe ampliarse a otro tipo de unidades diferentes a la moneda funcional para revelar una información de mejor funcionalidad respecto a temas importantes que se salen de la esfera puramente económica.

Una estructura contable de cuentas ambientales en términos físicos, explica la existencia de un conjunto de clases que refleja las categorías más significativas de un ente y comúnmente se le denomina Plan de Cuentas, que debe ser lo suficientemente consistente para ser aplicado a cualquier tipo de entidad y lo suficientemente flexible como para adaptarse a las necesidades de un ente en particular. Debe prestarse particular atención, la de establecer cuentas que tengan equivalencia entre distintos entes. La equivalencia mencionada anteriormente hace referencia a la relación que debe existir entre los elementos de una transacción, así, las salidas correspondientes a los productos elaborados por una empresa, deben relacionarse en una cuenta que sea equivalente a las entradas de productos utilizadas por otro ente.

El Plan de Cuentas es un instrumento concebido para armonizar y unificar el sistema contable de los principales sectores de la economía, además de mejorar las cualidades de la información contable y proporcionar beneficios a los usuarios, sirva para reconocer las relaciones entre la economía y el ambiente desde un punto de vista físico (flujos no monetarios), a través de un modelo contable ambiental que armonice y unifique con cierta flexibilidad la información. 


\section{ASPECTOS AMBIENTALES EN LA NORMA CONTABLE INTERNACIONAL}

La normativa contable emitida por el International Accounting Standards Board (IASB) referente a aspectos ambientales recomienda la inclusión de pasivosy activos ambientales en la información financiera de las empresas, así como también de contingencias y costos referidos al medio ambiente.

Los elementos relacionados directamente con la medida de la situación financiera son:

Patrimonio, es la parte residual de los activos de la entidad, una vez deducidos todos sus pasivos.

Pasivo, es una obligación presente de la entidad, surgida a raíz de sucesos pasados, al vencimiento de la cual, y para cancelarla, la entidad espera desprenderse de recursos que incorporan beneficios económicos.

Activo, es un recurso controlado por la entidad como resultado de sucesos pasados, del que la entidad espera obtener en el futuro, beneficios económicos.

Es interesante destacar dentro del concepto de activo la generación de beneficios económicos futuros dados por el potencial del mismo para contribuir directa o indirectamente, a los flujos de efectivo y de otros equivalentes al efectivo de la entidad.

Estos beneficios pueden llegar a la entidad por diferentes vías, un activo puede ser utilizado aisladamente, o en combinación con otros. En esta segunda situación es cuando aquellos activos incorporados con fines ambientales pueden tener relevancia.

$\mathrm{Al}$ respecto, los autores Pahlen R. y Campo A. (2009, p.11) señalan:

"Cabe preguntarnos si el reconocimiento contable de las ventajas competitivas que algunos recursos naturales aportan al aumento del patrimonio del ente, no deberían ser consideradas como tales en los informes a presentar a terceros, no olvidando la evolución del concepto de activo que hoy considera el Marco Conceptual, donde no se requiere propiedad sino solamente que se cumpla con el enfoque de los beneficios y riesgos que allíse indica. El hecho de ignorar el buen manejo de los recursos naturales por un ente, provocan un alejamiento a la estimación contable de un valor del ente y su capacidad para generar riqueza carecería de obje- tividad, pues presentaría sesgos hacia la subvaluación del patrimonio, cuando solo estaríamos reconociendo las contingencias negativas o las deudas emergentes de un inadecuado uso de esos recursos naturales".

En este sentido, la presentación de información ambiental dentro de los informes contables de uso externo genera un valor agregado a la hora de tomar decisiones.

\section{ACTIVOS Y COSTOS AMBIENTALES: NIC 16}

El Estado debe cumplir su rol con el cuidado y preservación del ambiente, aplicando los controles y legislación rigurosa para aquellas industrias que afectan el medio ambiente, induciendo a utilizar tecnología limpia, con materiales menos contaminantes y energía renovable.

Según Carvalho, G. en Panario Centeno, M.M., 2011 se reconoce a los activos ambientales como a "todos los bienes y derechos de la empresa, relacionados con la protección, preservación y recuperación ambiental y que están en condiciones de generar beneficios económicos futuros".

En el informe del grupo de trabajo intergubernamental de expertos en normas internacionales de contabilidad y presentación de informes de la secretaria de comercio y desarrollo de las Naciones Unidas, se plantean las siguientes definiciones:

Los costos ambientales abarcan los costos de las medidas adoptadas, o que deben adoptarse para la gestión ambientalmente responsable de los efectos ambientales de las actividades de una empresa, así como otros costos determinados por los objetivos y compromisos ambientales de la empresa.

La Conferencia de las Naciones Unidad sobre Comercio y Desarrollo (UNCTAD, 1998, p. 9) señala que los activos ambientales son costos ambientales capitalizados porque satisfacen los criterios para su reconocimiento como activos. Por capitalizar se entiende el registro de un costo ambiental como parte integrante de un activo conexo, o bien su reconocimiento como un activo separado, según proceda.

Asimismo, el informe establece el reconocimiento y activación de los costos ambientales si los

30/ QUipUiramavoc | Vol. 21(40) 2013 
mismos están relacionados, directa o indirectamente, con futuros beneficios económicos para la empresa resultantes de:

(a) un aumento de la capacidad o un mejoramiento de la seguridad o la eficiencia de otros activos de la empresa;

(b) una reducción o prevención de la contaminación ambiental que es probable que se produzca como resultado de operaciones futuras; o

(c) la conservación del medio ambiente.

Aunque esos costos, tal vez no determinen directamente un aumento de los beneficios económicos, pueden ser necesarios para que la empresa obtenga, o siga obteniendo en el futuro, beneficios económicos de sus otros activos.

Muchos costos ambientales no generan beneficios futuros o no guardan una relación suficientemente estrecha con beneficios futuros como para que se los pueda capitalizar o activar. Dichos costos deben ser tratados como gastos ambientales y en caso de estar relacionados con la actividad productiva de la empresa imputarlos a los bienes y servicios producidos por la empresa; como ejemplos de ambas situaciones podemos mencionar:

${ }^{*}$ Costos relativos al tratamiento de productos residuales.

${ }^{*}$ Costos de limpieza relacionados con actividades que se llevan a cabo durante el ejercicio en curso.

*Eliminación de los efectos de daños causados por la empresa.

*Actividades permanentes de administración ambiental.

*Auditoría ambiental.

${ }^{*}$ Multas por incumplimiento de normas ambiental.

${ }^{*}$ Indemnizaciones pagadas a terceras partes por daños ambientales.

La mayoría de las industrias que producen contaminación son aquellas relacionadas con el desarrollo económico del país, por ejemplo: las refinerías de petróleo; la siderurgia del hierro; la electricidad de origen térmico; las cementeras; las fábricas de celulosa; papel y cartón.
La influencia de este sector industrial sobre la política forestal (monocultivos de pino y eucalipto) o el "consumo" de bosques que implica la producción de cada tonelada de pasta de papel y las industrias químicas.

La necesidad de mejorar el desempeño ambiental, y con ello, el desempeño económico y social, hace pensar en el proceso productivo de un modo integral.

Fronti de García, L.ITCP, 2009, comenta, a partir de este enfoque surge el concepto de la producción limpia, basada en la prevención, los mecanismos de desarrollo limpio y las demás metodologías e innovaciones que persiguen como objetivo principal y último, el desarrollo sustentable concebido como la voluntad global de cuidar el futuro sustentable de la vida en la tierra, considerando las dimensiones económica, social, ambiental e institucional.

El proceso de producción limpia es una estrategia de gestión empresarial preventiva aplicada a los productos, procesos y organización del trabajo, cuyo objetivo es minimizar las emisiones tóxicas y de residuos, reduciendo así los riesgos para la salud humana y ambiental y elevando, simultáneamente, la competitividad organizacional.

La adquisición de bienes materiales destinados al uso por la organización, con el destino de proteger y preservar el medio ambiente puede implicar costos elevados que la empresa debe afrontar para corregiry mejorar las condiciones del medio ambiente conforme a los requerimientos actuales en materia de desarrollo sustentable. Dicha adquisición o construcción no responde a decisiones aisladas e insignificantes y mucho menos a importes no significantes, generalmente, son un punto clave de la estrategia ambiental de la empresa.

La incorporación de un activo de larga duración al patrimonio de un ente, así como, las mejoras sobre los mismos, se encuentra regulados por las normas contables profesionales.

La norma internacional emitida por el IASB, NIC 16 sobre propiedad, planta y equipo, prescribe su tratamiento contable y alude específicamente a ejemplos de índole ambiental. 


\section{CONCLUSIONES}

1. La inserción de la economía peruana en mercados internacionales y la firma de tratados de libre comercio, exige al gobierno un manejo sustentable de los recursos naturales y el ambiente, estableciendo políticas efectivas y económicamente eficientes para priorizar los problemas ambientales, con proyección hacia un mercado ambiental.

2. Se evidencia la necesidad e importancia de valorar desde un punto de vista económico los recursos naturales en nuestro país, de modo que permita la práctica de políticas efectivas y económicamente eficientes para un manejo sustentable del inventario de recursos naturales renovables y no renovables.

3. El proceso de valorar económicamente el medio ambiente se da a través de la medición y cuantificación de la calidad ambiental, permitiendo tomar decisiones estratégicas a través de la información contable ambiental.

4. La complejidad que presenta la valoración económica ambiental, hace necesaria la participación de los profesionales de las distintas disciplinas a fin establecer los lineamientos y métodos de valorización de bienes y servicios ambientales.

\section{REFERENCIAS BIBLIOGRÁFICAS}

1. Arias, G (2004). Análisis del impacto económico y social de las plantaciones forestales en Costa Rica. FUNDECOR. San José Costa Rica

2. D'onofrio, P (2006): "Tratamiento de los bienes de uso y sus erogaciones posteriores al reconocimiento inicial.

3. Barrantes, J (2005). Programa de Pago de Servicios Ambientales PSA, su evolución entre 19972004. ONF. México.

4. De Camino, R.( 2005). El manejo de bosques y plantaciones forestales al 2025 en Costa Rica. ¿Qué podemos esperar? "El sector forestal en Costa Rica, perspectivas al 2025. Costa Rica.

5. Fronti De Garcia, L. (2009): Sumideros y mecanismo de desarrollo limpio: una propuesta de desarrollo sostenible para países del MERCO-
SUR, documentos de trabajo de Contabilidad Patrimonial y Ambiental.

6. García F, Panario Centeno, María Marta (2011) Referencias a cuestiones ambientales en las normas internacionales de información financiera: Marco conceptual y bienes de uso, publicado en Enfoques 2012 (diciembre), 17-Normas Contables Internacionales.

7. Mark Fisher (2007) Las Leyes Espirituales de la Riqueza, editorial Urano.

8. Pahlen Acuña, R.; Campo, A. M.; Chaves, O.; Viegas, J. C. y Fronti de García, L. (2009) Contabilidad: pasado, presente y futuro. Editorial La Ley.

9. Quintero, M. (2006). Modelo de Optimización para Evaluación Ex Ante de Alternativas Productivas y Cuantificación de Externalidades Ambientales en Cuencas Andinas. Proyecto Regional Cuencas Andinas CONDESAN y Centro Internacional de Agricultura Tropical CIAT, Cali, Colombia.

10. United Nations Conference on Trade and Development -UNCTAD (1998) Contabilidad financiera y presentación de informes ambientales por las empresas. Grupo de Trabajo Intergubernamental de Expertos en Estándares Internacionales de Contabilidad y Presentación de Informes .Ginebra.

\section{REFERENCIAS HEMEROGRÁFICAS}

1. Autoridad Autónoma De Cuenca Hidrográfica Chira Piura y PDRS, GTZ. (2004).Diagnóstico Participativo con el Enfoque de Gestión del Riesgo de la Cuenca del Río Piura, Piura, Perú.

2. EDEPAS NORTE y Proyecto Regional Cuencas Andinas.(2007). Estudio Socio-Económico y de los Sistemas de Producción en la Cuenca del Río Jequetepeque, Cajamarca, Perú.

3. Machim, M. (2006). Valoración económica de los recursos naturales: Perspectivas a través de los diferentes enfoques de mercado. En: Revista Futuros No.13 Vol IV. 
4. Banco Interamericano de Desarrollo-BID. (2005). Estudio sobre inversión directa en negocios forestales sostenibles - proyecto atn/ np-8323-r. Índice de atracción a la inversión forestal (IAIF) Informe Final - rev. 01. División de administración de recursos naturales y medio ambiente departamento regional de operaciones. Curitiba - Brasil. Abril / 2005.

\section{REFERENCIAS ELECTRÓNICAS}

1. Vidal, S. Activos Ambientales, Visita 2-8-13 www. slideshare.net/wshy/anal - Colombia. 